\title{
Bifurcations and dynamics in convection with temperature-dependent viscosity in the presence of the $O(2)$ symmetry
}

\author{
J. Curbelo ${ }^{1,2}$ and A. M. Mancho ${ }^{1}$ \\ ${ }^{1}$ Instituto de Ciencias Matemáticas (CSIC-UAM-UCM-UC3M), Nicolás Cabrera, 13-15, 28049 Madrid, Spain \\ ${ }^{2}$ Departamento de Matemáticas, Facultad de Ciencias, Universidad Autónoma de Madrid, 28049 Madrid, Spain
}

(Received 16 May 2013; published 14 October 2013)

\begin{abstract}
We focus on the study of a convection problem in a two-dimensional setup in the presence of the $\mathrm{O}(2)$ symmetry. The viscosity in the fluid depends on the temperature as it changes its value abruptly in an interval around a temperature of transition. The influence of the viscosity law on the morphology of the plumes is examined for several parameter settings, and a variety of shapes ranging from spout- to mushroom-shaped are found. We explore the impact of the symmetry on the time evolution of this type of fluid, and we find solutions which are greatly influenced by its presence: at a large aspect ratio and high Rayleigh numbers, traveling waves, heteroclinic connections, and chaotic regimes are found. These solutions, which are due to the presence of symmetry, have not been previously described in the context of temperature-dependent viscosities. However, similarities are found with solutions described in other contexts such as flame propagation problems or convection problems with constant viscosity also in the presence of the $\mathrm{O}(2)$ symmetry, thus confirming the determining role of the symmetry in the dynamics.
\end{abstract}

DOI: 10.1103/PhysRevE.88.043005

PACS number(s): 47.20.Ky, 05.45.-a

\section{INTRODUCTION}

This paper addresses the numerical study of convection at infinite Prandtl number in fluids in which viscosity strongly depends on temperature in the presence of the $\mathrm{O}(2)$ symmetry. Convection in fluids with temperature-dependent viscosity is of interest because of its importance in engineering and geophysics. Linear and quadratic dependencies of the viscosity on temperature have been discussed [1-4], but in order to address the Earth's upper mantle convection, in which viscosity contrasts are of several orders of magnitude, a stronger dependence with temperature must be considered. This problem has been approached both in experiments [5-8] and in theory [9-14]. In these contexts, the dependence of viscosity with temperature is expressed by means of an Arrhenius law. In [10], the exponential dependence is discussed as an approach to the Arrhenius law by means of a Taylor series around a reference temperature. This is also called the Frank-Kamenetskii approximation (see [15]). Viscosity has also been considered when it depends on other magnitudes such as depth [16,17], a combination of both depth and temperature [17], or pressure [18]. However, it is commonly accepted $[18,19]$ that in the Earth's interior, viscosity depends most significantly on temperature. The usual approach in numerical models of the mantle $[12,17,20]$ is to consider constant thermal conductivity. This approach has also been verified in fluid experiments seeking to model mantle convection [7]. However, studies also exist in which variations on thermal conductivity are considered [21-23].

Here, we focus on the study of a fluid in which the viscosity changes abruptly in a temperature interval around a temperature of transition. This defines a phase change over a mushy region, which expresses the melting of minerals or other components. Melting and solidification processes are important in magma chamber dynamics $[24,25]$, in volcanic conduits [26,27], in the formation of chimneys in mushy layers [28], in metal processing in industry (see, for example, [29]), etc. In phase transitions, other fluid properties in addition to viscosity may change abruptly, such as density or thermal diffusivity. However, in this study we consider solely the study of effects due to the variability of viscosity, since consideration of the effect of simultaneous variations on all the properties prevents a focused understanding of the exact role played by each one of these properties. Viscosity is a measure of fluid resistance to gradual deformation, and in this sense very viscous fluids are more likely to behave rigidly when compared to less viscous fluids. When examining the proposed transition with temperature, we focus on the global fluid motion when some parts of it tend to be more rigid than others. Disregarding the variations on density in this transition moves us away from instabilities caused by abrupt density changes such as the Rayleigh-Taylor instability, in which a denser fluid over a lighter one tends to penetrate it by forming a fingering pattern. A recent article by Ulvrová et al. [30] deals with a problem similar to ours, but takes into account variations both in density and in viscosity. Thermal conductivity effects are related to the relative importance of heat advection versus diffusion. In this way, diffusive effects are important at large conductivity, while heat advection by fluid particles is dominant at low conductivity. The contrasts arising from these variations are beyond the scope of our work and thus are disregarded here.

This paper addresses the convection of a two-dimensional (2D) fluid layer with temperature-dependent viscosity and periodic boundary conditions possessing the $O(2)$ symmetry. The motivation arises from the fact that symmetric systems typically exhibit more complicated bifurcations than nonsymmetric systems and introduce conditions and degeneracies in bifurcation analysis. There exist numerous novel dynamical phenomena whose existence is fundamentally related to the presence of symmetry [31-34]. Solutions related to the presence of symmetry include rotating waves [35], modulated waves [36,37], slow "phase" drifts along directions of broken symmetry [38], and stable heteroclinic cycles $[37,39,40]$. $\mathrm{SO}(2)$ symmetry is present in problems described by the Navier-Stokes $[41,42]$ or the Kuramoto-Sivashinsky $[40,43]$ equations with periodic boundary conditions, since the 
equations are invariant under translations and the boundary conditions do not break this invariance. Additionally, if the reflection symmetry exists, the full symmetry group is the $\mathrm{O}(2)$ group. While in classical convection problems (with constant viscosity), the study of the solutions and bifurcations in the presence of symmetries has been the object of much attention [44-51], its counterpart in fluids with viscosity depending on temperature has received less consideration. Our 2D physical setup is idealized with respect to realistic geophysical flows occurring in the Earth's interior, as these are three-dimensional (3D) flows moving in spherical shells [52,53]. Under these conditions, the symmetry present in the problem is formed by all the orientation-preserving rigid motions of $\mathbb{R}^{3}$ that fix the origin, which is the $\mathrm{SO}(3)$ group [54-56]. The effects of the Earth's rotation are negligible in this respect and do not break this symmetry, since the high viscosity of the mantle makes the Coriolis number insignificant. The link between our simplified problem and these realistic setups is that the $\mathrm{O}(2)$ symmetry is isomorphic to the rotations along the azimuthal coordinate, which form a closed subgroup of $\mathrm{SO}(3)$. Additionally, the $\mathrm{O}(2)$ symmetry is present in systems with cylindrical geometry, which provide an idealized setting for volcanic conduits and magma chambers. $\mathrm{SO}(2)$ symmetry is also present in 3D flows moving in spherical shells which rotate around an axis.

In this way, specific symmetry-related solutions found in our setting are expected to be present in these other contexts. The interest in 2D numerical studies for representing 3D time-dependent thermal convection with constant viscosity has been addressed in [57]. The authors report that, in turbulent regimes at high Rayleigh numbers, the flow structure and global quantities such as the Nusselt number and the Reynolds number show a similar behavior in $3 \mathrm{D}$ and $2 \mathrm{D}$ simulations as far as high values of the Prandtl number are concerned. In some sense, these results suggest that our simulations might be illustrative for the $3 \mathrm{D}$ case, since although they are far from a turbulent regime and do not correspond to the case of constant viscosity, they have been performed according to the infinite Prandtl number approach. In this article we show that typical solutions of systems with symmetries, as previously reported in diverse contexts [37,40,58], could be also present in mantle convection and magma-related problems. We report the presence of traveling waves and limit cycles near heteroclinic connections after a Hopf bifurcation. We do this by means of bifurcation analysis techniques and direct numerical simulations of the full partial differential equation system.

The article is organized as follows: In Sec. II, we formulate the problem, providing the description of the physical setup, the basic equations, and the boundary conditions. In Sec. III we present the viscosity law under consideration and discuss several limits in which previously studied dependencies are recovered. Section IV summarizes the numerical methods used to sketch an outlook of the solutions displayed by the system. Section V discusses the solutions obtained for a broad parameter set. Finally, Sec. VI presents the conclusions.

\section{FORMULATION OF THE PROBLEM}

As shown in Fig. 1 we consider a fluid layer, placed in a 2D container of length $L$ ( $x$ coordinate) and depth $d$ ( $z$ coordinate).

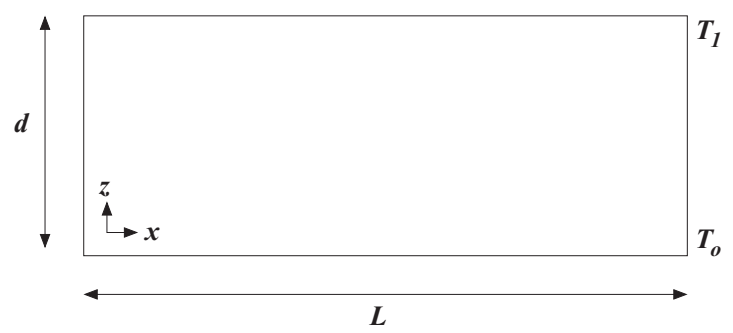

FIG. 1. Problem setup. A 2D container of length $L$ and depth $d$ with periodic lateral boundary conditions. The bottom plate is at temperature $T_{0}$ and is rigid, while at the upper plate the temperature is $T_{1}\left(T_{1}<T_{0}\right)$ and free slip.

The bottom plate is at temperature $T_{0}$ and the upper plate is at $T_{1}$, where $T_{1}=T_{0}-\Delta T$ and $\Delta T$ is the vertical temperature difference, which is positive, i.e., $T_{1}<T_{0}$.

The magnitudes involved in the equations governing the system are the velocity field $\mathbf{u}=\left(u_{x}, u_{z}\right)$, the temperature $T$, and the pressure $P$. The spatial coordinates are $x$ and $z$ and the time is denoted by $t$. Equations are simplified by invoking the Boussinesq approximation, where the density $\rho$ is considered as constant everywhere except in the external forcing term, where a dependence on temperature is assumed, as follows: $\rho=\rho_{0}\left[1-\alpha\left(T-T_{1}\right)\right]$. Here $\rho_{0}$ is the mean density at temperature $T_{1}$ and $\alpha$ is the thermal expansion coefficient.

The equations are expressed with magnitudes in dimensionless form after rescaling as follows: $\left(x^{\prime}, z^{\prime}\right)=(x, z) / d$, $t^{\prime}=\kappa t / d^{2}, \mathbf{u}^{\prime}=d \mathbf{u} / \kappa, P^{\prime}=d^{2} P /\left(\rho_{0} \kappa v_{0}\right)$, and $\theta^{\prime}=(T-$ $\left.T_{1}\right) /(\Delta T)$. Here, $\kappa$ is the thermal diffusivity and $v_{0}$ is the maximum viscosity of the fluid, which is the viscosity at temperature $T_{1}$. After the domain is rescaled, $\Omega_{1}=[0, L) \times$ $[0, d]$ is transformed into $\Omega_{2}=[0, \Gamma) \times[0,1]$, where $\Gamma=L / d$ is the aspect ratio. The system evolves according to the momentum and the mass balance equations, as well as to the energy conservation principle. The nondimensional equations are (after dropping the primes in the fields)

$$
\begin{gathered}
\nabla \cdot \mathbf{u}=0, \\
\frac{1}{\operatorname{Pr}}\left(\partial_{t} \mathbf{u}+\mathbf{u} \cdot \nabla \mathbf{u}\right) \\
=\operatorname{Ra} \theta \vec{e}_{3}-\nabla P+\operatorname{div}\left(\frac{\nu(\theta)}{v_{0}}\left(\nabla \mathbf{u}+(\nabla \mathbf{u})^{T}\right)\right), \\
\partial_{t} \theta+\mathbf{u} \cdot \nabla \theta=\Delta \theta .
\end{gathered}
$$

Here, $\vec{e}_{3}$ represents the unitary vector in the vertical direction, $\mathrm{Ra}=d^{4} \alpha g \Delta T /\left(v_{0} \kappa\right)$ is the Rayleigh number, $g$ is the acceleration of gravity, and $\operatorname{Pr}=\nu_{0} / \kappa$ is the Prandtl number. Typically for rocks $\operatorname{Pr}$ is very large, since they present low thermal conductivity (approximately $10^{-6} \mathrm{~m}^{2} / \mathrm{s}$ ) and very large viscosity (of the order of $10^{20} \mathrm{~N} \mathrm{~s} / \mathrm{m}^{2}$ ) [19]. Thus, for the problem under consideration, Pr can be considered as infinite and the left-hand-side term in (2) can be made equal to zero. The viscosity $v(\theta)$ is a smooth positive bounded function of $\theta$, which in our setup represents a transition in the fluid, due for instance to the melting of minerals caused by an abrupt change in viscosity at a certain temperature. This is discussed in detail in the following section. 


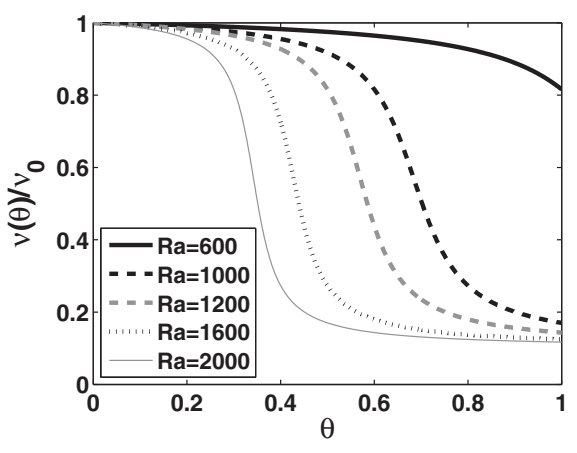

(a)

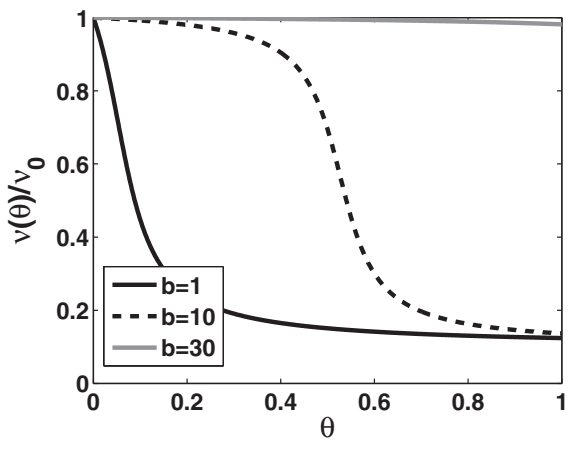

(b)

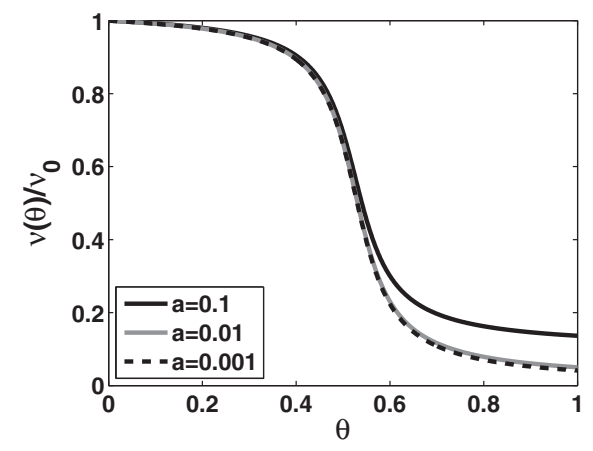

(c)

FIG. 2. Representation of the arctangent viscosity law vs the dimensionless temperature for different parameters values; (a) $b=10, a=0.1$, and different Ra values; (b) $a=0.1, \mathrm{Ra}=1300$, and different $b$ values; (c) $b=10, \mathrm{Ra}=1300$, and different $a$ values.

For the boundary conditions, we consider that the bottom plate is rigid and that the upper surface is nondeformable and free slip. The dimensionless boundary conditions are expressed as

$$
\theta=1, \mathbf{u}=\overrightarrow{0} \text { on } z=0 \text { and } \theta=\partial_{z} u_{x}=u_{z}=0 \text { on } z=1 .
$$

Lateral boundary conditions are periodic. Jointly with Eqs. (1)-(3), these conditions are invariant under translations along the $x$ coordinate, which introduces the symmetry $\mathrm{SO}(2)$ into the problem. In convection problems with constant viscosity, reflection symmetry $x \rightarrow-x$ is also present insofar as the fields are conveniently transformed as follows: $\left(\theta, u_{x}, u_{z}, p\right) \rightarrow$ $\left(\theta,-u_{x}, u_{z}, p\right)$. In this case, the $\mathrm{O}(2)$ group expresses the full symmetry of the problem. The new terms introduced by the temperature-dependent viscosity in the current setup equation (2) maintain the reflection symmetry, and the symmetry group is $\mathrm{O}(2)$.

\section{THE VISCOSITY LAW}

We consider that the viscosity depends on temperature and that it changes more or less abruptly at a certain temperature interval centered at a temperature of transition. This is expressed with an arctangent law, which reads as follows:

$$
v(T)=A_{1} \arctan \left\{\beta\left[\left(T-T_{1}\right)-b\right]\right\}+A_{2} .
$$

The parameter $\beta$ controls how abrupt the transition of the viscosity with temperature is. Very high $\beta$ values imply that the viscosity transition occurs within a very narrow temperature gap, while a finite and not too large $\beta$ value indicates that the phase change happens over a mushy region of finite thickness [30]. For the results reported in this article, we have fixed $\beta=0.9$. As $\beta$ is fixed, the viscosity transition always occurs in a temperature interval with constant thickness $\Delta \theta \sim 0.23$. The temperature at which the transition occurs is controlled by $b$. The constants $A_{1}$ and $A_{2}$ are adjusted by imposing that at the reference temperature $T_{1}$ the viscosity law (5) must be $v_{0}$. On the other hand, in the limit $T \gg T_{1}$, for instance $T-T_{1}=2500$, the viscosity is fixed to a fraction $a$ of the viscosity $v_{0}$. These conditions supply the system

$$
\begin{aligned}
v_{0} & =A_{1} \arctan (-\beta b)+A_{2}, \\
v_{0} a & =A_{1} \arctan [\beta(2500-b)]+A_{2},
\end{aligned}
$$

which has the solution

$$
\begin{aligned}
& A_{1}=\frac{v_{0}(1-a)}{\arctan (-\beta b)-\arctan [\beta(2500-b)]}, \\
& A_{2}=v_{0}-A_{1} \arctan (-b \beta) .
\end{aligned}
$$

In dimensionless form, the viscosity law becomes

$$
\frac{\nu(\theta)}{v_{0}}=C_{1} \arctan [\beta(\operatorname{Ra} \theta \mu-b)]+C_{2},
$$

where $C_{1}=A_{1} / \nu_{0}$ and $C_{2}=A_{2} / \nu_{0}$. In this expression, Ra is the Rayleigh number and $\theta$ is the dimensionless temperature, which takes values between 0 at the upper surface and 1 at the bottom. The parameter $\mu$, defined as $\mu=v_{0} \kappa /\left(d^{3} \alpha g\right)$, is in this study fixed to $\mu=0.0146$. The parameter $a$ is related to the inverse of the maximum viscosity contrast on the fluid layer, although the viscosity $v_{0} a$ may not correspond to any element of the fluid layer. For instance, Fig. 2(a) shows the viscosity variation with temperature for different Rayleigh numbers at $a=0.1$ and $b=10$. It is observed that, at low $\mathrm{Ra}, \mathrm{Ra}=600$, the viscosity is almost uniform in the fluid layer, and it is only beyond $\mathrm{Ra}=1000$ that the sharp change in the viscosity is perceived. Figure 2(b) shows the effect of varying $b$ at $\mathrm{Ra}=1300$ and $a=0.1$. If $b$ is as small as 1 , the transition occurs close to $\theta=1$ and most of the layer has low viscosity, while if $b$ is very large at this Ra number most of the fluid has constant viscosity $v_{0}$. It is interesting to relate the viscosity law as represented in these figures with the linear stability analysis of a fluid layer with constant viscosity $v_{0}$, as presented in Fig. 3. In this figure, one may observe that the critical value of $\mathrm{Ra}$ is approximately $\mathrm{Ra}_{c} \sim 1100$. On the other hand, in Fig. 2(b) one may observe that if $b$ is large, the viscosity near the critical Rayleigh number is almost constant across the fluid layer. In this case, the phase transition is noticed in the fluid at large $\mathrm{Ra}$, well above $\mathrm{Ra}=1300$, in a convection state in which vigorous plumes are already formed, as may be deduced from Fig. 2(a). Figure 3(a) confirms that at this limit the instability threshold of the conductive state remains unchanged with respect to that obtained with constant viscosity. On the other hand, if $b$ is small, changes in the 


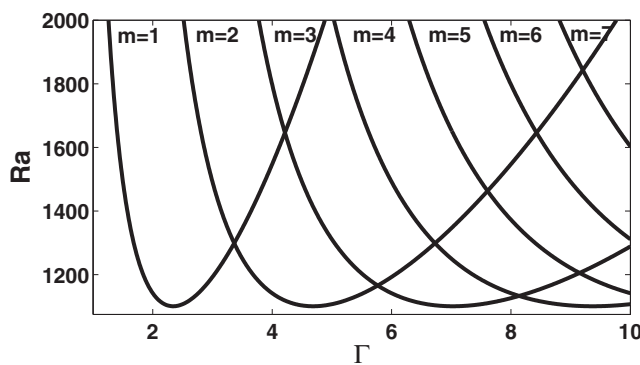

(a)

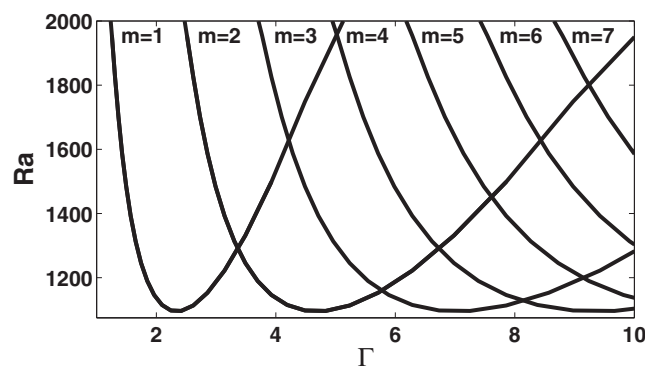

(b)

FIG. 3. Critical instability curves of the Rayleigh number, Ra, vs the aspect ratio $\Gamma$ at different wave numbers $m$. The results are for a fluid layer (a) with constant viscosity and (b) with temperature-dependent viscosity $\mu=0.0146$ for $a=0.1$ and $b=30$.

fluid viscosity are noticed at low Ra values - below the critical threshold of a fluid with constant viscosity - and in this case the instability threshold of the conductive state is affected by the phase transition. This is illustrated, for instance, in Figs. 2(a) and 3(b). For $b=10$ and $a=0.1$, the changes in the viscosity across the fluid layer are noticed from $\mathrm{Ra}=800$ onward, which is below the instability threshold obtained for constant viscosity. In this case, the instability thresholds for the conductive solution are as those displayed in Fig. 4, and thus the phase transition is perceived from the beginning by weakly convective states.

We now discuss the relation between the arctangent law and an Arrhenius-type law frequently used in the literature to model mantle convection problems. This viscosity law is expressed according to $[19,59]$ as

$$
v(\theta)=v_{0} \exp \left[\frac{E^{*}}{\bar{R} \Delta \theta}\left(\frac{1}{\theta+t_{1}}-\frac{1}{1+t_{1}}\right)\right],
$$

where $E^{*}$ is the activation energy, $\bar{R}$ is the universal gas constant, $\Delta \theta$ is the temperature drop across the fluid layer, and $t_{1}$ is the surface temperature divided by the temperature drop across the layer. Figure 5 represents the viscosity (7) versus the dimensionless temperature for $\frac{E^{*}}{\bar{R} \Delta \theta}=0.25328$ and $t_{1}=0.1$ as considered by [59]. Additionally, several arctangent laws with different $b$ values are displayed. In this representation,

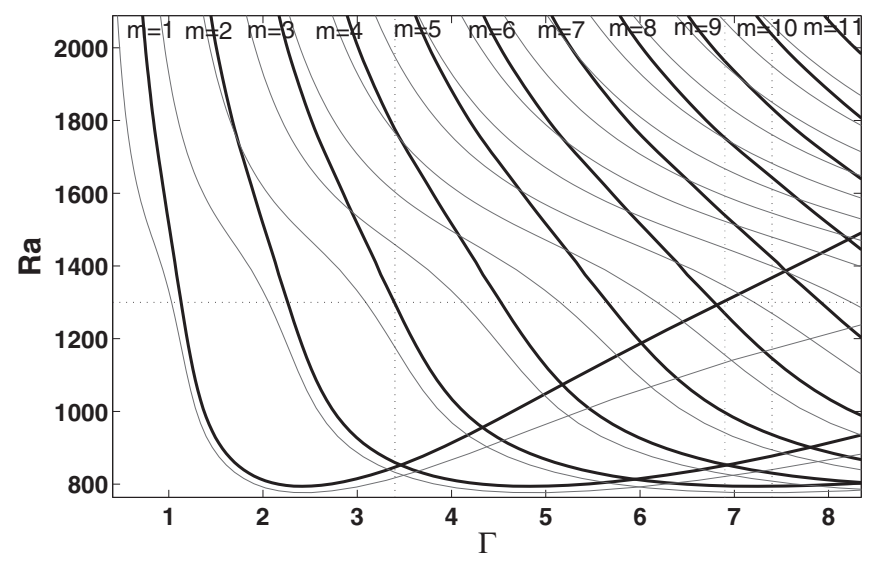

FIG. 4. Critical instability curves of the Rayleigh number, Ra, vs the aspect ratio $\Gamma$ at different wave numbers $m$. The results are for a fluid layer with temperature-dependent viscosity $\mu=0.0146$ and $b=10$ and $a=0.1$ (thick line) or $a=0.01$ (thin line). one may observe great similitude between the Arrhenius law and the arctangent law for $b=1$. At larger $b$ values, the decay rate between viscosities is still similar to an Arrhenius law; however, temperature intervals exist with approximately constant viscosities $v_{0}$ and $v_{0} a$.

One of the effects of the viscosity contrasts in the fluid motion is that if they are very large, as achieved for instance with the exponential or the Arrhenius law, they lead to a stagnant lid convection regime [12,60,61], in which there exists a nonmobile cap where heat is dissipated mainly by conduction over a convecting flow. In [30,62] a similar stagnant regime is obtained for a viscosity law similar to the one presented in this section. In our setting, we have considered a free-slip boundary at the top boundary; thus quiescence is not imposed. This condition enables us to consider spontaneous transitions from stagnant to nonstagnant regimes.

\section{NUMERICAL METHODS}

Analysis of the solutions to the problem described by Eqs.(1)-(3) and boundary conditions (4) is assisted by timedependent numerical simulations and bifurcation techniques such as branch continuation. As highlighted in [63,64], the combination of both techniques provide a thorough insight into the solutions observed in the system. A full discussion on the spectral numerical schemes used is given in [63]. For

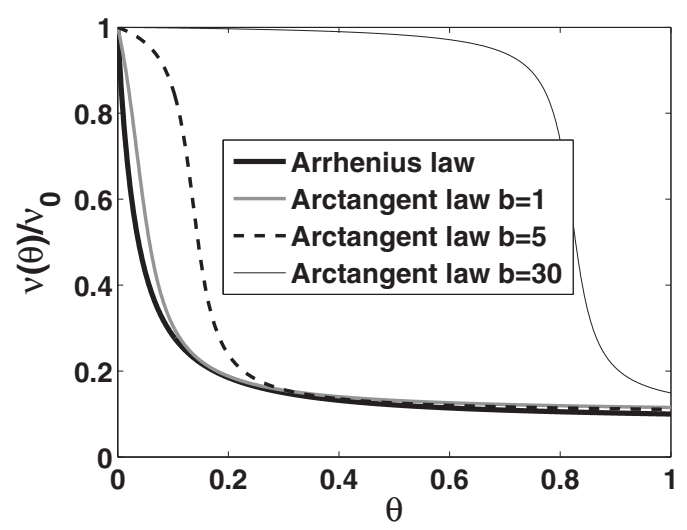

FIG. 5. The law of the viscosity dependent on temperature used in [59] with a viscosity contrast of a factor of 10 against the arctangent law (6) with parameters $b=1,5,10,30, \mathrm{Ra}=2500$, and $a=0.1$. 
completeness, we now summarize the essential elements of the numerical approach.

\section{A. Stationary solutions and their stability}

The simplest stationary solution to the problem described by Eqs. (1)-(3) with boundary conditions (4) is the conductive solution which satisfies $\mathbf{u}_{c}=0$ and $\theta_{c}=-z+1$. This solution is stable only for a range of vertical temperature gradients which are represented by small enough Rayleigh numbers. Beyond the critical threshold $\mathrm{Ra}_{c}$, a convective motion settles in and new structures are observed which may be either time dependent or stationary. In the latter case, the stationary equations, obtained by canceling the time derivatives in the system (1)-(3), are satisfied by the bifurcating solutions. At the instability threshold of the conductive state, the growing solutions are periodic and correspond to sine or cosine eigenfunctions with wave number $m$. Figures 3 and 4 display the critical instability curves for different $m$ values as a function of the aspect ratio. The new solutions depend on the external physical parameters, and new critical thresholds exist at which stability is lost, thereby giving rise to new bifurcated structures. These solutions are numerically obtained by using an iterative Newton-Raphson method. This method starts with an approximate solution at step $s=0$, to which is added a small correction (denoted by a tilde):

$$
\left(\mathbf{u}^{s}+\tilde{\mathbf{u}}, \theta^{s}+\tilde{\theta}, P^{s}+\tilde{P}\right) .
$$

These expressions are introduced into the system (1)-(3), and after canceling the nonlinear terms in tilde, the following equations are obtained:

$$
\begin{gathered}
0=\nabla \cdot \tilde{\mathbf{u}}+\nabla \cdot \mathbf{u}^{s} \\
0=-\partial_{x} \tilde{P}-\partial_{x} P^{s}+\frac{1}{v_{0}}\left[L_{11}\left(\theta^{s}, u_{x}^{s}, u_{z}^{s}\right)+L_{12}\left(\theta^{s}\right) \tilde{u}_{x}\right. \\
\left.+L_{13}\left(\theta^{s}\right) \tilde{u}_{z}+L_{14}\left(\theta^{s}, u_{x}^{s}, u_{z}^{s}\right) \tilde{\theta}\right] \\
0=-\partial_{z} \tilde{P}-\partial_{z} P^{s}+\frac{1}{v_{0}}\left[L_{21}\left(\theta^{s}, u_{x}^{s}, u_{z}^{s}\right)+L_{22}\left(\theta^{s}\right) \tilde{u}_{x}\right. \\
\left.+L_{23}\left(\theta^{s}\right) \tilde{u}_{z}+\left(L_{24}\left(\theta^{s}, u_{x}^{s}, u_{z}^{s}\right)+\mathrm{Ra}\right) \tilde{\theta}\right] \\
0=\tilde{\mathbf{u}} \cdot \nabla \theta^{s}+\mathbf{u}^{s} \cdot \nabla \tilde{\theta}+\mathbf{u}^{s} \cdot \nabla \theta^{s}-\Delta \tilde{\theta}-\Delta \theta^{s} .
\end{gathered}
$$

Here, $L_{i j}(i=1,2, j=1,2,3,4)$ are linear operators with nonconstant coefficients, which are defined as follows:

$$
\begin{gathered}
L_{11}\left(\theta, u_{x}, u_{z}\right)=2 \partial_{\theta} v(\theta) \partial_{x} \theta \partial_{x} u_{x}+v(\theta) \Delta u_{x} \\
+\partial_{\theta} v(\theta) \partial_{z} \theta\left(\partial_{x} u_{z}+\partial_{z} u_{x}\right) \\
L_{12}(\theta)=2 \partial_{\theta} v(\theta) \partial_{x} \theta \partial_{x}+v(\theta) \Delta+\partial_{\theta} v(\theta) \partial_{z} \theta \partial_{x} \\
L_{13}(\theta)=\partial_{\theta} v(\theta) \partial_{z} \theta \partial_{x} \\
L_{14}\left(\theta, u_{x}, u_{z}\right)=2 \partial_{\theta} v(\theta) \partial_{x} u_{x} \partial_{x}+2 \partial_{\theta \theta}^{2} v(\theta) \partial_{x} \theta \partial_{x} u_{x} \\
+\partial_{\theta} v(\theta) \Delta u_{x}+\left(\partial_{x} u_{z}+\partial_{z} u_{x}\right)\left(\partial_{\theta} v(\theta) \partial_{z}\right. \\
\left.+\partial_{\theta \theta}^{2} v(\theta) \partial_{z} \theta\right) \\
L_{21}\left(\theta, u_{x}, u_{z}\right)=2 \partial_{\theta} v(\theta) \partial_{z} \theta \partial_{z} u_{z}+v(\theta) \Delta u_{z} \\
+\partial_{\theta} v(\theta) \partial_{x} \theta\left(\partial_{z} u_{x}+\partial_{x} u_{z}\right)
\end{gathered}
$$

$$
L_{22}(\theta)=\partial_{\theta} v(\theta) \partial_{x} \theta \partial_{z},
$$

$$
L_{23}\left(\theta, u_{x}, u_{z}\right)=2 \partial_{\theta} v(\theta) \partial_{z} \theta \partial_{z}+v(\theta) \Delta+\partial_{\theta} v(\theta) \partial_{x} \theta \partial_{z},
$$

$$
\begin{aligned}
& L_{24}\left(\theta, u_{x}, u_{z}\right) \\
& =2 \partial_{\theta} v(\theta) \partial_{z} u_{z} \partial_{z}+2 \partial_{\theta \theta} v(\theta) \partial_{z} \theta \partial_{z} u_{z}+\partial_{\theta} v(\theta) \Delta u_{z} \\
& \quad+\left(\partial_{z} u_{x}+\partial_{x} u_{z}\right)\left(\partial_{\theta} v(\theta) \partial_{x}+\partial_{\theta \theta} v(\theta) \partial_{x} \theta\right) .
\end{aligned}
$$

The unknown fields $\tilde{\mathbf{u}}, \tilde{P}$, and $\tilde{\theta}$ are found by solving the linear system with the boundary conditions

$$
\tilde{\theta}=0, \quad \tilde{\mathbf{u}}=\overrightarrow{0} \text { on } z=0 \text { and } \tilde{\theta}=\partial_{z} \tilde{u_{x}}=\tilde{u_{z}}=0 \text { on } z=1 \text {. }
$$

Then the new approximate solution $s+1$ is set to

$$
\mathbf{u}^{s+1}=\mathbf{u}^{s}+\tilde{\mathbf{u}}, \theta^{s+1}=\theta^{s}+\tilde{\theta}, P^{s+1}=P^{s}+\tilde{P} .
$$

The whole procedure is repeated for $s+1$ until a convergence criterion is fulfilled. In particular, we consider that the $l^{2}$ norm of the computed perturbation should be less than $10^{-9}$.

The study of the stability of the stationary solutions under consideration is addressed by means of a linear stability analysis. Now perturbations are added to a general stationary solution, labeled with superindex $b$ :

$$
\begin{gathered}
\mathbf{u}(x, z, t)=\mathbf{u}^{b}(x, z)+\tilde{\mathbf{u}}(x, z) e^{\lambda t}, \\
\theta(x, z, t)=\theta^{b}(x, z)+\tilde{\theta}(x, z) e^{\lambda t}, \\
P(x, z, t)=P^{b}(x, z)+\tilde{P}(x, z) e^{\lambda t} .
\end{gathered}
$$

The sign in the real part of the eigenvalue $\lambda$ determines the stability of the solution: if it is negative, the perturbation decays and the stationary solution is stable, while if it is positive the perturbation grows over time and the conductive solution is unstable. The linearized equations are

$$
\begin{gathered}
0=\nabla \cdot \tilde{\mathbf{u}}, \\
0=-\partial_{x} \tilde{P}+\frac{1}{v_{0}}\left[L_{12}\left(\theta^{b}\right) \tilde{u}_{x}+L_{13}\left(\theta^{b}\right) \tilde{u}_{z}\right. \\
\left.+L_{14}\left(\theta^{b}, u_{x}^{b}, u_{z}^{b}\right) \tilde{\theta}\right], \\
0=-\partial_{z} \tilde{P}+\frac{1}{v_{0}}\left[L_{22}\left(\theta^{b}\right) \tilde{u}_{x}+L_{23}\left(\theta^{b}\right) \tilde{u}_{z}\right. \\
\\
\left.+\left(L_{24}\left(\theta^{b}, u_{x}^{b}, u_{z}^{b}\right)+\mathrm{Ra}\right) \tilde{\theta}\right], \\
0 \tilde{\mathbf{u}} \cdot \nabla \theta^{b}+\mathbf{u}^{b} \cdot \nabla \tilde{\theta}+\mathbf{u}^{b} \cdot \nabla \theta^{b}-\Delta \tilde{\theta}+\lambda \tilde{\theta},
\end{gathered}
$$

where the operators $L_{i j}$ are the same as those defined in Eqs. (13)-(20). Equations (25)-(28) jointly with its boundary conditions [identical to (21)] define a generalized eigenvalue problem.

The unknown fields $Y$ of the stationary (9)-(12) and eigenvalue problems (25)-(28) are approached by means of 
a spectral method according to the expansion

$$
\begin{aligned}
Y(x, z)= & \sum_{l=1}^{\lceil L / 2\rceil} \sum_{m=0}^{M-1} b_{l m}^{Y} T_{m}(z) \cos [(l-1) x] \\
& +\sum_{l=2}^{\lceil L / 2\rceil} \sum_{m=0}^{M-1} c_{l m}^{Y} T_{m}(z) \sin [(l-1) x] .
\end{aligned}
$$

In this notation, $\lceil\cdot\rceil$ represents the nearest integer toward infinity. Here $L$ is an odd number as justified in [63]. $4 \times L \times M$ unknown coefficients exist and these are determined by a collocation method in which equations and boundary conditions are imposed at the collocation points $\left(x_{j}, z_{i}\right)$,

$$
\text { uniform grid: } \quad x_{j}=(j-1) \frac{2 \pi}{L}, j=1, \ldots, L \text {, }
$$

Gauss-Lobatto: $z_{i}=\cos \left[\left(\frac{i-1}{M-1}-1\right) \pi\right], i=1, \ldots, M$, according to the rules detailed in [63]. Expansion orders $L$ and $M$ are taken to ensure accuracy on the results: details on their values are provided in Sec. V.

\section{B. Time-dependent schemes}

Together with boundary conditions (4), the governing equations (1)-(3) define a time-dependent problem for which we propose a temporal scheme based on a spectral spatial discretization analogous to that proposed in the previous section. As before, expansion orders $L$ and $M$ are such that they ensure accuracy of the results, and details on their values are given in the following section. To integrate in time, we use a third-order multistep scheme. In particular, we use a backward differentiation formula (BDF), adapted for use with a variable time step. The variable time step scheme controls the step size according to an estimated error $E$ for the fields. The error estimation $E$ is based on the difference between the solutions obtained with third- and second-order schemes. The result of an integration at time $n+1$ is accepted if $E$ is below a certain tolerance. Details of the step adjustment are found in [63].

BDFs are a particular case of multistep formulas which are implicit; thus the BDF scheme implies solving at each time step the problem (see [65])

$$
\begin{gathered}
0=\nabla \cdot \mathbf{u}^{n+1}, \\
0=\operatorname{Ra} \theta^{n+1} \overrightarrow{e_{3}}-\nabla P^{n+1} \\
+\operatorname{div}\left(\frac{v\left(\theta^{n+1}\right)}{v_{0}}\left(\nabla \mathbf{u}^{n+1}+\left(\nabla \mathbf{u}^{n+1}\right)^{T}\right)\right), \\
\partial_{t} \theta^{n+1}=-\mathbf{u}^{n+1} \cdot \nabla \theta^{n+1}+\Delta \theta^{n+1},
\end{gathered}
$$

where $\partial_{t} \theta^{n+1}$ is replaced by a backward differentiation formula.

In [63], it has been proved that instead of solving the fully implicit scheme (30)-(32), a semi-implicit scheme can produce results with a similar accuracy and less computation time. The semi-implicit scheme approaches the nonlinear terms in Eqs. (30)-(32) if one assumes that the solution at time $n+1$ is a small perturbation $\tilde{Z}$ of the solution at time $n$; thus, $\mathbf{z}^{n+1}=\mathbf{z}^{n}+\tilde{Z}$. Once linear equations for $\tilde{Z}$ are derived, the equations are rewritten by replacing $\tilde{Z}=\mathbf{z}^{n+1}-\mathbf{z}^{n}$. The solution is obtained at each step by solving the resulting linear equation for variables in time $n+1$.

\section{RESULTS}

\section{A. Exploration of stationary solutions in the parameter space}

In this section we explore how stationary solutions obtained at a low aspect ratio $\Gamma=3.4$ for the system (1)-(3) depend on the parameters $a$ and $b$ of the viscosity law (6). We examine the shape and structure of the plumes in a range of Rayleigh numbers from $\mathrm{Ra}=2500$ to $\mathrm{Ra}=3500$.

We first consider that the parameter $b$ is large: for instance, as large as 30. In this case, Fig. 2(b) confirms that at the instability threshold the viscosity across the fluid layer is almost constant and equal to $v_{0}$, no matter what the value of $a$ may be. Thus, the viscosity transition becomes evident in the fluid once convection has settled in at Ra values well above the instability threshold. Figure 6(a) shows the plume pattern observed at $\mathrm{Ra}=2500$ for $a=0.1$; although values $a=0.01$ and $a=0.001$ are not displayed, they provide a very similar output. The plume is spout-shaped, with the tail of the plume nearly as large as the head. In the pattern, the two black contour lines mark temperatures between which the viscosity decays most rapidly. These correspond to the transition region in which the gradient of the viscosity law (6) is large. Thus one of the contours, the coldest one, fits the temperature $\theta_{1}$ at which the viscosity has decayed by $5 \%$ from the maximum, i.e., $v=0.95 v_{0}$, while the second addresses $\theta_{2}=\theta_{1}+\Delta \theta$ with temperature increment $\Delta \theta=0.23$. The maximum viscosity decay rate always takes place at a constant temperature increment, since the decay rate of the law (6), $\beta$, is the same throughout this study. At larger Rayleigh numbers, $\mathrm{Ra}=3500$, Fig. 6(b) shows that the head of the plume becomes more prominent. A comparison between Fig. 6(b) and Fig. 6(c) indicates that the large viscosity contrast favors the formation of a balloon-shaped plume, with a thinner tail and more prominent and rounded head. As regards the velocity fields, none of these patterns develop a stagnant lid at the surface for any of the viscosity contrasts $a$ considered, even though the upper part corresponds to the region with maximum viscosity. This result is dissimilar to what is obtained in [30,62]. In [62] it is argued that the cause of these differences could be attributed to the transition sharpness controlled by $\beta$, which in this work has been considered to be smoother. Additionally, the results reported in [30] are obtained at larger viscosity contrasts, and the fact that these need to be large enough for the development of a stagnant lid has been addressed.

We now consider that the parameter $b$ is small. As explained in Sec. III, in this case the viscosity transition occurs at low $\mathrm{Ra}$, below the instability threshold of the fluid with constant viscosity $v_{0}$. As low viscosity also implies diminishing the critical Rayleigh number, the overall effect is that for small $b$ the instability threshold is below that with constant viscosity $v_{0}$, and the phase transition is perceived by weakly convective states. Figure 6(d) shows the structure of the plume obtained 


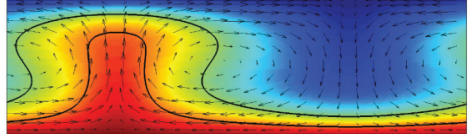

(a) $b=30, \mathrm{Ra}=2500, a=0.1$

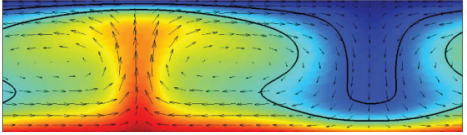

(d) $b=10, \mathrm{Ra}=2500, a=0.1$

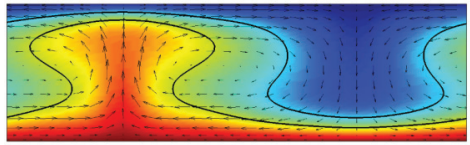

(g) $b=17, \mathrm{Ra}=2500, a=0.1$

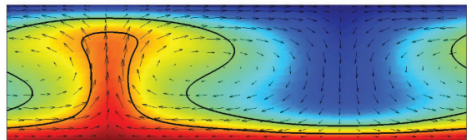

(b) $b=30, \mathrm{Ra}=3500, a=0.1$

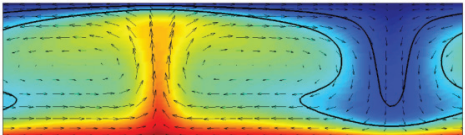

(e) $b=10, \mathrm{Ra}=3500, a=0.1$

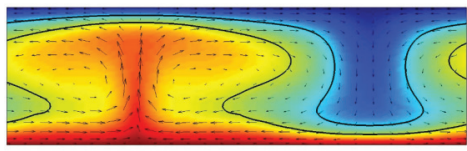

(h) $b=17, \mathrm{Ra}=2500, a=0.001$

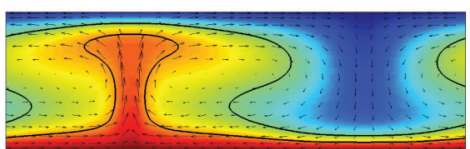

(c) $b=30, \mathrm{Ra}=3500, a=0.001$

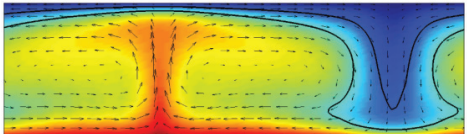

(f) $b=10, \mathrm{Ra}=2500, a=0.001$

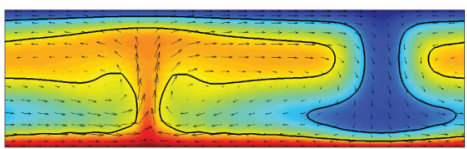

(i) $b=17, \mathrm{Ra}=3000, a=0.001$

FIG. 6. (Color online) Plumes obtained for several values of the viscosity parameter $b$. The arrows indicate the velocity field, while the contour colors represent the temperature ranging from hot (bottom plate) to cold (upper plate). The two black contour lines indicate the temperatures between which viscosity decays most rapidly.

for $b=10$ and $a=0.1$ at $\mathrm{Ra}=2500$. The head tends to be spread over a wide area and the viscosity transition occurs at cold fluid zones away from the main plume. This pattern is rather similar to those obtained with $b=5$ or $b=1$, except that for smaller $b$ values the tail of the plume tends to be thinner. Increasing Ra makes the tail of the plume thinner and spreads the head of the plume in the upper part, as reflected in Fig. 6(e). On the other hand, high Ra values shift the viscosity transition toward colder temperature contours. As expected from the viscosity law (6), there is no Rayleigh number at which the whole fluid layer is "melted," since this law always imposes that a transition occurs across the fluid layer. Figure 6(f) reports the effect of diminishing the viscosity contrast $a$ to $a=0.001$ at $\mathrm{Ra}=2500$. A mushroom-shaped plume with a thin tail and prominent head is observed. As before, none of these solutions develop a stagnant lid at the surface for any of the examined viscosity contrasts $a$.

Intermediate values such as $b=17$ interpolate these extreme patterns. Figure $6(\mathrm{~g})$ shows the evolution from Fig. 6(d) to Fig. 6(a) in which the black contour lines indicating the position of maximum viscosity decay converge toward the ascending plume boundary, thus highlighting its shape. The head of the plume shrinks and the tail strengthens. Diminishing $a$ to the contrast 0.001 transforms the structure into a balloon-shaped plume [Fig. 6(h)], while an increase in Ra spreads the head of the plume in the upper fluid toward a mushroom-shaped plume.

The structure of the observed plumes as a spout, balloon, or mushroom shape follows the schematic profiles reported in [59]. In the limit of low $b$, our viscosity law-as reported in Sec. III-converges toward the Arrehnius law used by these authors, and the plume shapes reported there are similar to ours. However, a detailed comparison between both works is not possible as unlike these authors we include the Rayleigh number in the viscosity law, since this provides a better expression of the realistic situation in which the increment of the Rayleigh number is performed by increasing the temperature differences between the bottom and upper surfaces. Other viscosity laws, such as the exponential law reported in [63], provide different plume structures, which are mainly spout-shaped.

The results reported in this section are obtained with expansions $(L \times M=37 \times 44)$, except that in Fig. 6(c), which corresponds to $(L \times M=47 \times 42)$, similarly to what is reported in [63]. The validity of these expansions is decided by ensuring that it provides accuracy in the eigenvalue along the neutral direction due to the $\mathrm{SO}(2)$ symmetry, which is always 0 . This eigenvalue is lost if the expansions employed are insufficiently large, because badly resolved basic states present noisy structures either at the fields themselves or at their derivatives, and both contribute to the stability problem (26)-(28).

\section{B. Bifurcation diagrams and time-dependent solutions}

Solutions to the system (1)-(3) experience bifurcations depending on the aspect ratio and on the Rayleigh number. We now describe how these solutions vary along the dotted lines enhanced in Fig. 4 for parameters $\mu=0.0146$ and $b=10$. We consider for $a$ the choices 0.1 and 0.01 .

Figure 7 shows the branch bifurcation diagram as a function of the aspect ratio for $\mathrm{Ra}=1300$ and $a=0.1$. Branches are obtained by representing along the vertical axis the sum of the absolute value of two relevant coefficients in the expansion of the temperature field, $b_{11}^{\theta}$ and $b_{12}^{\theta}$. Solid lines stand for stable branches, while dashed lines are the unstable ones. The horizontal line at $\left|b_{11}^{\theta}\right|+\left|b_{12}^{\theta}\right|=1$ corresponds to the trivial conductive solution. At a low aspect ratio, the stable branch is that with wave number $m=1$, and at a higher aspect ratio the stable solutions increase their wave number to $m=2$ and $m=3$. The unstable branch ending up with a saddle-node bifurcation and connecting the $m=1$ with the $m=2$ branch corresponds to a mixed mode.

Stationary stable and unstable solutions, obtained at the positions indicated by arrows, are pictured. No stagnant lid 


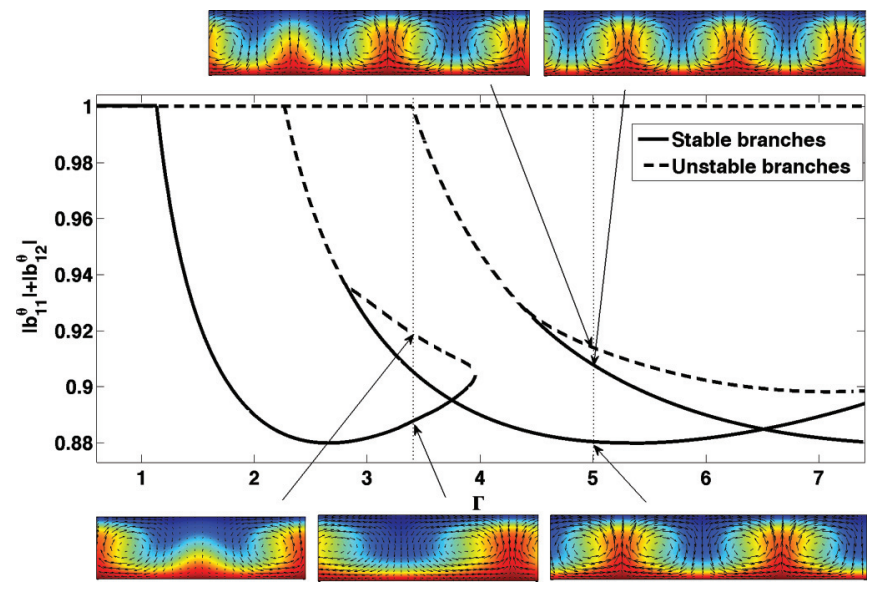

FIG. 7. (Color online) Bifurcation diagram as a function of the aspect ratio at $\mathrm{Ra}=1300$ for a fluid with viscosity dependent on temperature ( $b=10, a=0.1$ ). Stationary solutions are displayed at different Ra numbers, which are highlighted by vertical lines. The arrows tag the branch points corresponding to the disclosed patterns. The dashed branches are unstable, while the solid ones are stable.

appears at the surface for any of the aspect ratios considered. The expansion orders required by this figure to ensure accuracy are not the same along all branches. We have guaranteed that for successive order expansions the amplitude values displayed on the vertical axis of the bifurcation diagrams are preserved. A rule of thumb is that high modes obtained at larger aspect ratios require higher expansions. Thus while for mode $m=1$ expansions $(L \times M=37 \times 44)$ are sufficient, for $m=2$ and $m=3$ at larger aspect ratios expansions are increased up to $(L \times M=61 \times 44)$.

Bifurcations are further analyzed at three different aspect ratios as a function of the Rayleigh number. Among the many possible choices for the aspect ratios, we consider occurrences at which the existence of solutions related to symmetries are found, such choices thereby serving our purpose of highlighting the importance of symmetries in fluids with viscosity dependent on temperature. Figure 8 represents the branching obtained at $\Gamma=3.4$ for $a=0.1$. The pictured plumes, which are computed for a rather low Rayleigh number, $\mathrm{Ra}=1500$, are spout-shaped, with the tail of the plume nearly as large as the head. As already reported in the previous section, for increasing Ra, plumes become balloon-shaped and beyond that mushroom-shaped. No stagnant lid is observed at any Rayleigh number. Several branches are distinguished. The branch related to mode $m=1$ arises at the lowest Rayleigh number and is stable in the whole range displayed. Mode $m=2$ emerges at $\mathrm{Ra} \sim 860$ from the unstable conductive solution through an unstable branch, which becomes stable through a pitchfork bifurcation at $\mathrm{Ra} \sim 890$. Results at this aspect ratio are obtained with expansions $(L \times M=37 \times 44)$.

This simple diagram with simple stationary solutions obtained at a low aspect ratio is in contrast to those with more complex solutions obtained at a larger aspect ratio. Figure 9 represents the bifurcations obtained at $\Gamma=6.9$ as a function of Ra for $a=0.01$. Figure 9(a) examines the Ra interval from 800 to 1300 . In this range several stationary solutions, both stable and unstable, are portrayed.

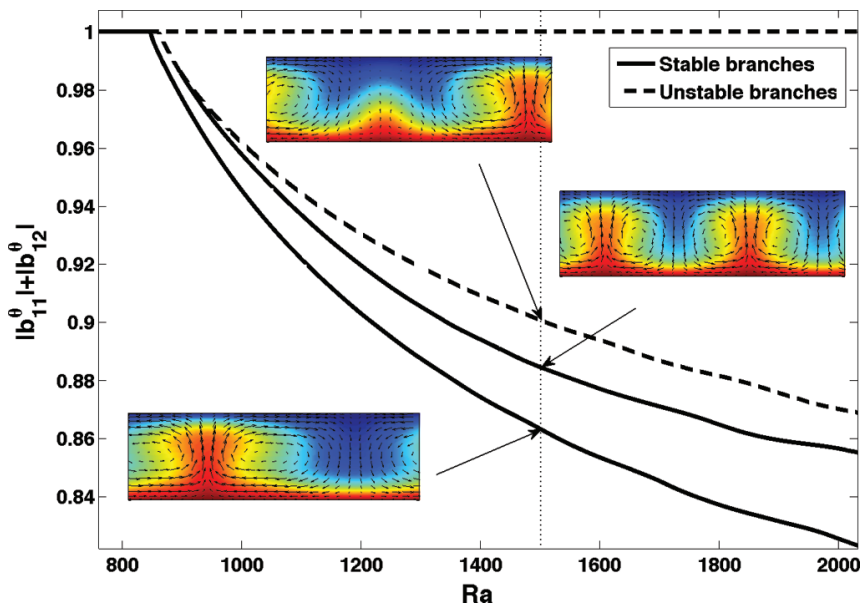

FIG. 8. (Color online) Bifurcation diagram as a function of the Rayleigh number for a fluid with viscosity dependent on temperature ( $b=10, a=0.1)$ at $\Gamma=3$.4. Stationary solutions are displayed at the Ra number, which is highlighted with the vertical line. The arrows tag the branch points corresponding to the disclosed patterns. The dashed branches are unstable, while the solid ones are stable.

At $\mathrm{Ra} \sim 1290$, a Hopf bifurcation occurs at the branch of mode $m=3$ [see Fig. 9(b)]. After the bifurcation, a traveling wave is found, as illustrated in the phase portrait represented at $\mathrm{Ra}=1300$. The solution evolves in time by traveling toward the left. This breaks the symmetry $x \rightarrow-x$. However, the right traveling solution obtained by the symmetry transformation also exists, as expected from equivariant bifurcation theory [31]. See [66] for further details. The presence of traveling waves after a Hopf bifurcation has been reported in diverse contexts in the presence of the $\mathrm{O}(2)$ symmetry $[31,37,40,58]$, and here they are reported in the context of convection with variable viscosity. At larger Rayleigh numbers, up to $\mathrm{Ra} \sim 1320$, the traveling wave persists, while its frequency increases. A stable fixed point with wave number $m=3$ is found in the range $\mathrm{Ra} \sim 1340-1380$. A limit cycle appears at around $\mathrm{Ra} \sim 1400$. In this regime, the time-dependent solution consists of plumes that weakly oscillate in the horizontal direction around their vertical axis of symmetry. See [66] for further details. Close to Ra $\sim 1416$, a stable branch of fixed points emerges, which is visualized at $\mathrm{Ra} \sim 1525$. It shows the presence of plumes that are nonuniformly distributed along the horizontal coordinate: two close plumes, which are asymmetric around their vertical axis, and a third one that maintains its symmetry. None of the described solutions develop stagnant lids at the surface. At low Rayleigh numbers [i.e., Fig. 9(a)] results are obtained with expansions $(L \times M=47 \times 44)$, while for higher Rayleigh numbers [i.e., Fig. 9(b)] results are obtained with expansions $(L \times M=61 \times 44)$.

Figure 10 shows the bifurcation diagram obtained at $\Gamma=7.4$ as a function of Ra for $a=0.1$. The mode $m=3$ branch, marked with a solid black line, emerges at $\mathrm{Ra} \sim 794$. Figure 10(b) shows that at $R \sim 2190$ the branch undergoes a Hopf bifurcation. Beyond this point, solutions embedded in a projection over the coefficient space are represented at the $R$ values marked with vertical dotted lines. A limit cycle is observed at $\mathrm{Ra}=2210$ just above the bifurcation 


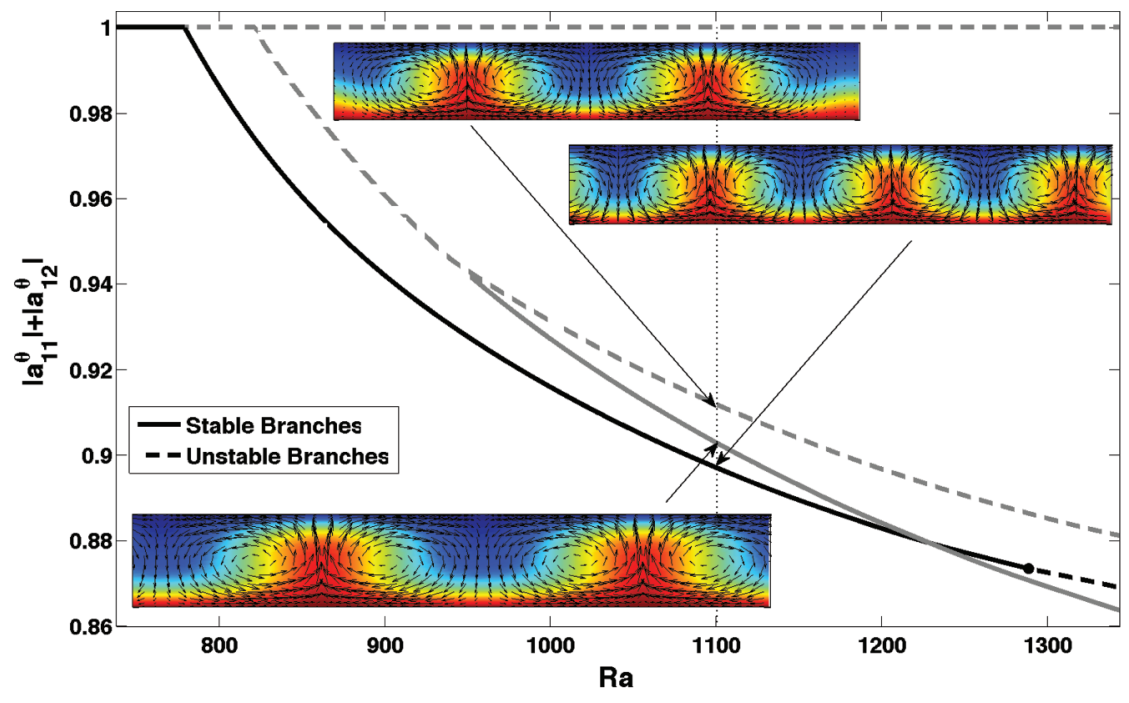

(a)

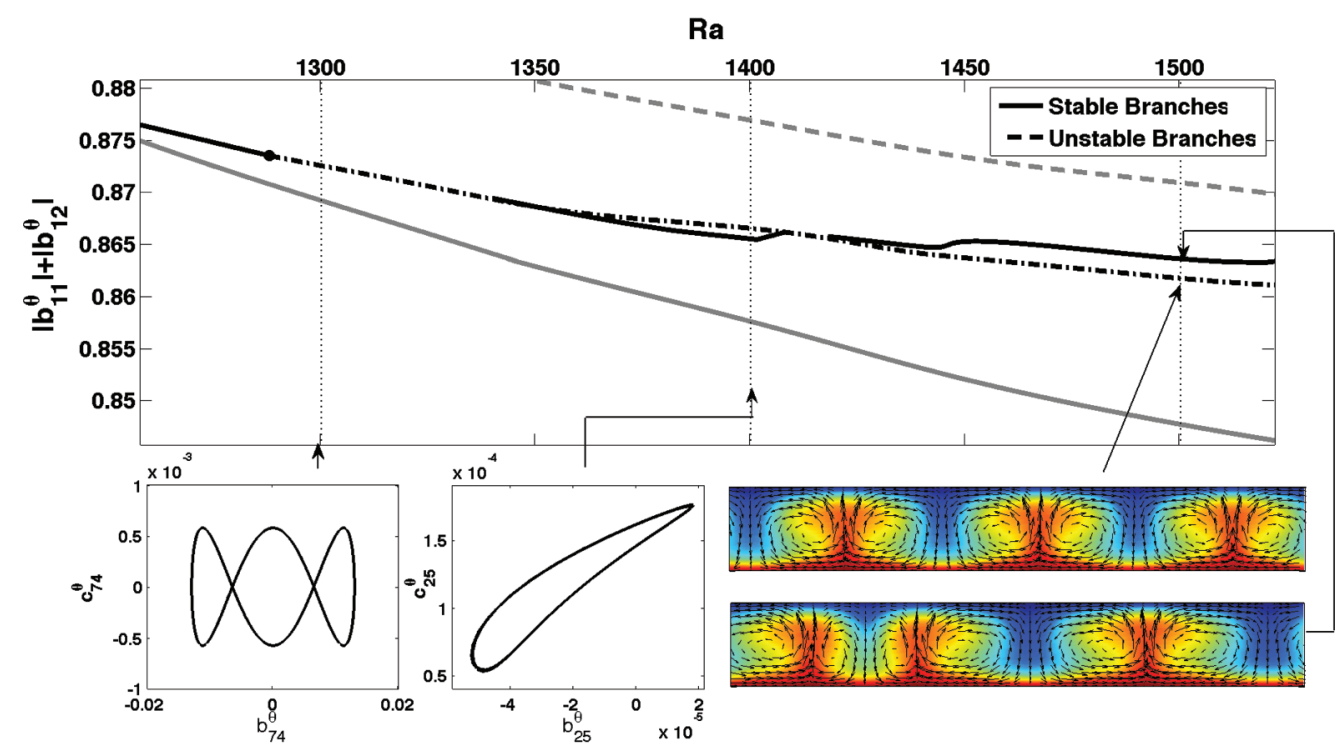

(b)

FIG. 9. (Color online) Bifurcation diagrams as a function of the Rayleigh number for a fluid with viscosity dependent on temperature ( $b=10, a=0.01)$ at $\Gamma=6.9$. The dashed branches correspond to stationary unstable solutions, while solid branches correspond to stationary stable ones. The gray lines indicate spatial patterns with period 2, while the black ones are for period-3 patterns. (a) Rayleigh number in the range 800-1300. Stationary solutions are displayed at the Rayleigh number highlighted with the vertical line. Arrows tag the branch points corresponding to the disclosed patterns; (b) Rayleigh number in the range 1250-1500. Stationary solutions are displayed at the Rayleigh number, which is highlighted by the vertical line. The arrows tag the branch points corresponding to the disclosed patterns. Two additional vertical lines highlight Rayleigh numbers of 1300 and 1400 at which time-dependent solutions are found. These are displayed as a time series projected onto the coefficient space (for a description see the text and [66]).

point. Its projection over the coefficient space displays a point at every time step of the time series. The solution appears to reside in the neighborhood of a heteroclinic connection between two fixed points as it evolves into a quasistationary regime —-near the large density of points — followed by a rapid transition to a new quasistationary regime. The two fixed points between which the solution oscillates are similar to the nonuniformly distributed plumes described in the previous paragraph (see [66] for further details). A solution is found at $\mathrm{Ra}=2300$ that has a time dependence in which the block of plumes shifts irregularly along the horizontal direction, toward both the left and the right (see [66]). For increasing
Rayleigh numbers, the horizontal motion persists, but the oscillation becomes more regular and pattern displacements along the $x$ coordinate are gradually reduced. This is verified through simulations at $\mathrm{Ra}=2350$ and at $\mathrm{Ra}=2400$ (see [66]). The diagram displayed in Fig. 10(a) shows a gray solid line associated with a mode $m=2$ stable branch that emerges by means of a saddle-node bifurcation jointly with an unstable branch. An irregular pattern obtained at $\mathrm{Ra}=1800$ for the unstable branch is included in this diagram. Once again, none of the solutions described at this aspect ratio have a stagnant lid at the surface. Results in this figure are obtained with different order expansions. At low Rayleigh number expansions 


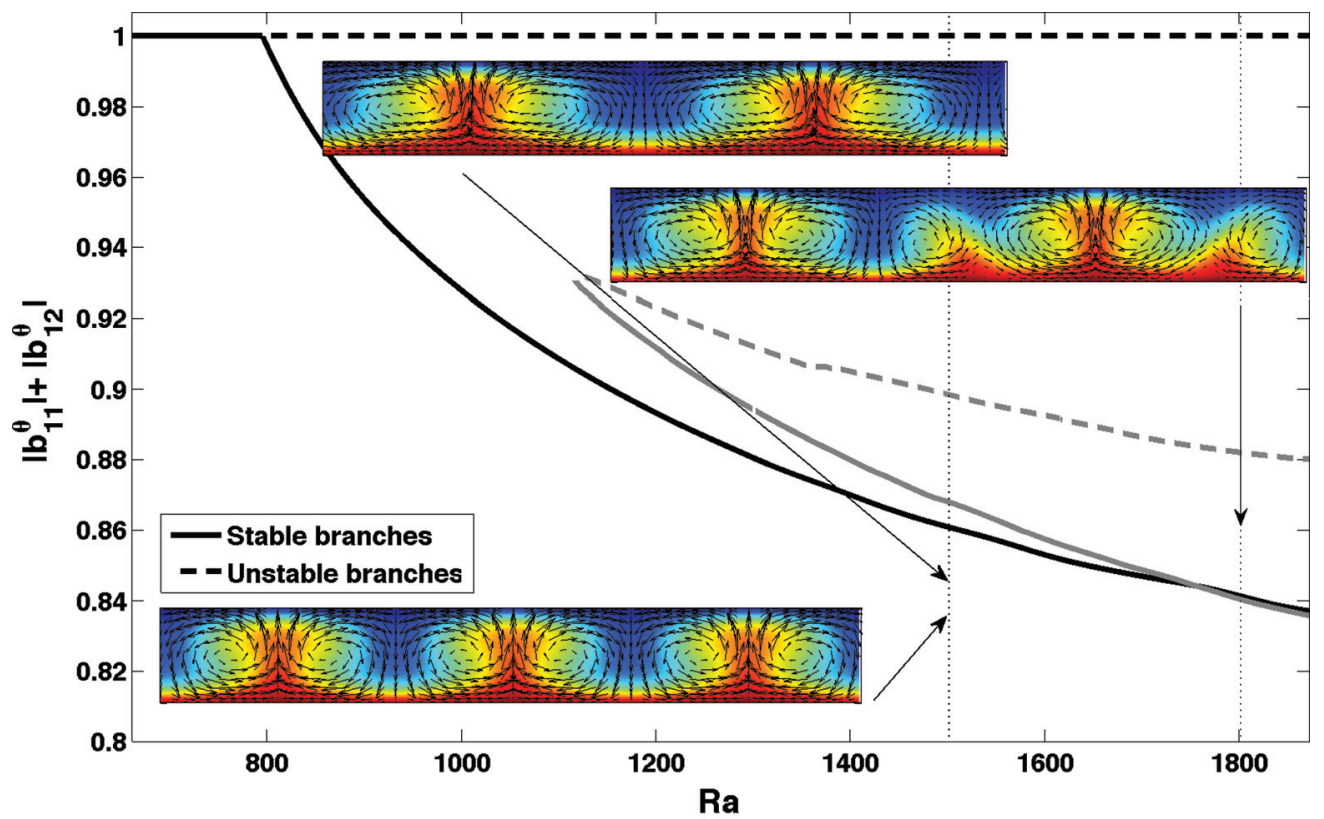

(a)

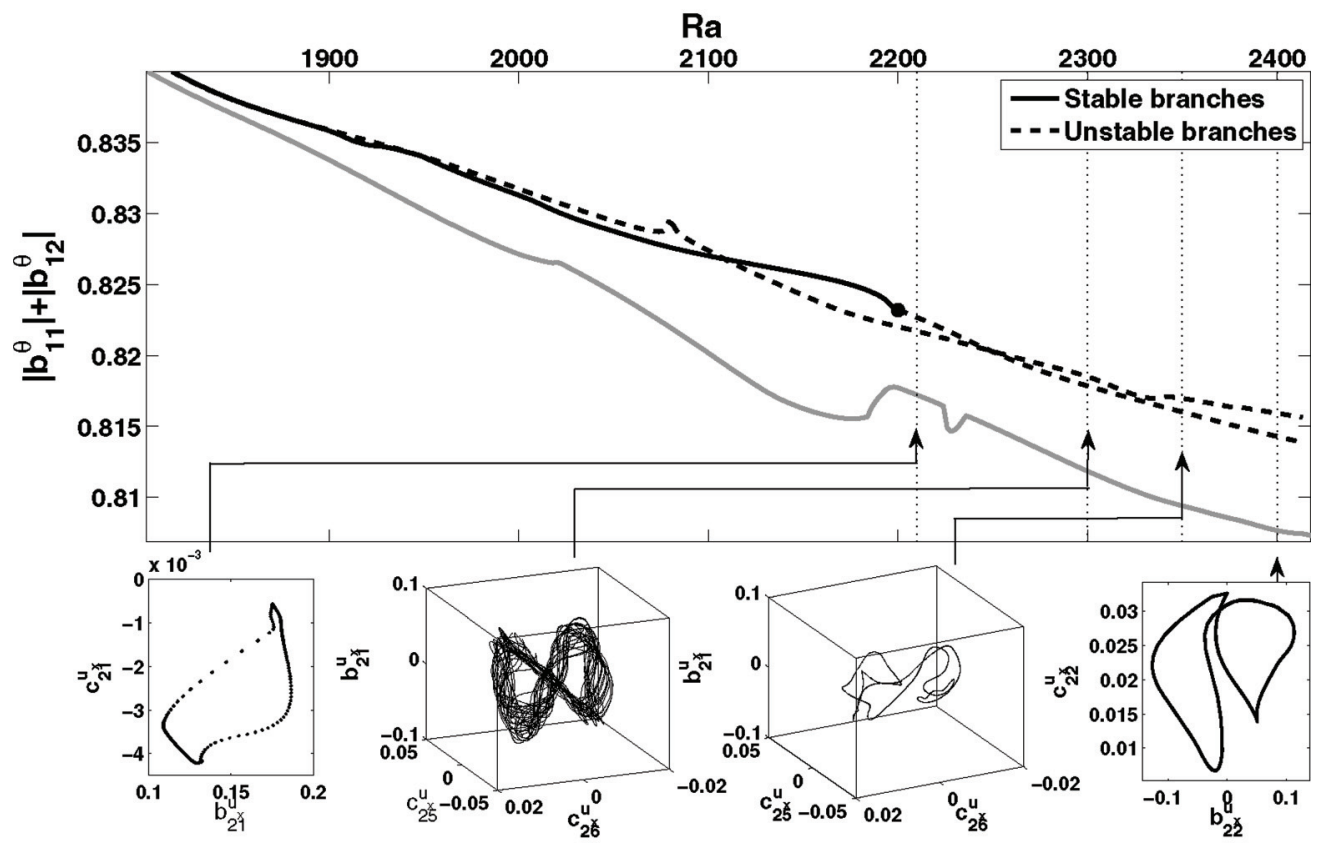

(b)

FIG. 10. (Color online) Bifurcation diagrams as a function of the Rayleigh number for a fluid with viscosity dependent on temperature $(b=10, a=0.1)$ at $\Gamma=7.4$. The dashed branches correspond to stationary unstable solutions, while solid branches correspond to stationary stable ones. The gray lines stand for spatial patterns with period 2 while the black ones are for period-3 patterns. (a) Rayleigh number in the range 700-1800. Stationary solutions are displayed at the Rayleigh number, which is highlighted by the vertical line. The arrows tag the branch points corresponding to the disclosed patterns; (b) Rayleigh number in the range 1800-2500. Vertical lines highlight the Rayleigh numbers at which time-dependent solutions are found. These are 2210, 2300, 2350, and 2400. These are displayed as a time series projected onto the coefficient space (for a description see the text and [66]).

$(L \times M=47 \times 42)$ are sufficient while for higher Rayleigh numbers they are increased up to $(L \times M=61 \times 44)$ and even to $(L \times M=101 \times 44)$.

The time-dependent solutions reported in Figs. 9 and 10 in many respects resemble those described for the KuramotoSivashinsky (KS) equation $[40,67]$ in the presence of the
$\mathrm{O}(2)$ symmetry, in which the presence of traveling waves and heteroclinic cycles are also reported. The $\mathrm{KS}$ equation is proposed in order to describe thermal diffusive instabilities in flame fronts [68], and while apparently this setting is rather different from ours, the similitude between solutions suggest that the abrupt changes in the viscosity could define 
a similar kind of front to those observed in flame propagation phenomena. On the other hand, similar solutions have been found in 3D convection with constant viscosity in the presence of the $\mathrm{O}(2)$ symmetry $[49,50]$, thus confirming the determining role of the symmetry in the dynamics.

\section{CONCLUSIONS}

In this article we have addressed the study of a convection problem with temperature-dependent viscosity in the presence of the $\mathrm{O}(2)$ symmetry. In particular, the considered viscosity law represents a viscosity transition at a certain temperature interval around a temperature of transition. This is a problem of great interest for its many applications in geophysical and industrial flows and in this work the focus has been on exploring the impact of symmetry on the solutions displayed by the system.

Our results report the influence on parameters $a$ and $b$ of the viscosity law on the morphology of the plumes at a low aspect ratio $(\Gamma=3.4)$. It is shown that if the temperature of transition is well above the instability threshold of a fluid with constant viscosity $v_{0}$, i.e., $b$ is large, plumes tend to be thicker and show spout-like shapes. Increasing the Rayleigh number induces their evolution toward balloon-shaped plumes, and this effect is more pronounced for high viscosity contrasts (small $a$ ). At low $b$ values plumes are thinner, and the head of the plume tends to spread in a mushroom-like shape in the upper part of the fluid.

We explore bifurcations for both a fixed $\mathrm{Ra}$ as a function of the aspect ratio and at three fixed aspect ratios as a function of the Ra. No stagnant lid regime is observed in any of the physical conditions analyzed. Among the stationary solutions obtained along the bifurcation branches, one of the more interesting stable patterns consists of the nonuniformly distributed plumes that break symmetry along their vertical axis.

We also find that, for the higher Rayleigh numbers explored, at a high aspect ratio several rich dynamical phenomena appear. As already reported in classical convection problems, we find dynamical phenomena fundamentally related to the presence of symmetry, such as traveling waves, oscillating solutions in the neighborhood of heteroclinic connections, and chaotic regimes characterized by "phase" drifts along the horizontal direction linked to the $\mathrm{SO}(2)$ symmetry.

\section{ACKNOWLEDGMENTS}

We are grateful to CESGA and to CCC of Universidad Autónoma de Madrid for computing facilities. This research is supported by the Spanish Ministry of Science under Grants No. MTM2008-03754 and No. MTM2011-26696 and MINECO: ICMAT Severo Ochoa project SEV-2011-0087.
[1] E. Palm, T. Ellingsen, and B. Gjevik, J. Fluid Mech. 30, 651 (1967).

[2] L. Richardson and B. Straughan, Acta Mech. 97, 41 (1993).

[3] J. I. Diaz and B. Straughan, Continuum Mech. Thermodyn. 16, 347 (2004).

[4] A. Vaidya and R. Wulandana, Math. Methods Appl. Sci. 29, 1555 (2006).

[5] F. M. Richter, H. C. Nataf, and S. F. Daly, J. Fluid Mech 129, 173 (1983).

[6] F. Capone and M. Gentile, Acta Mech. 107, 53 (1994).

[7] J. R. Booker, J. Fluid Mech. 76, 741 (1976).

[8] D. B. White, J. Fluid Mech. 191, 247 (1988).

[9] M. Ogawa, G. Schubert, and A. Zebib, J. Fluid Mech. 233, 299 (1991).

[10] A. Bottaro, P. Metzener, and M. Matalon, Phys. Fluids 4, 655 (1992).

[11] J. T. Ratcliff, P. J. Tacley, G. Schubert, and A. Zebib, Phys. Earth Planet. Inter. 102, 201 (1997).

[12] L. N. Moresi and V. S. Solomatov, Phys. Fluids 7, 2154 (1995).

[13] V. S. Solomatov, Phys. Earth Planet. Inter. 200-201, 63 (2012).

[14] V. S. Solomatov and A. C. Barr, Phys. Earth Planet. Inter. 165, 1 (2007).

[15] G. R. Fulford and P. Broadbridge, Industrial Mathematics, Australian Mathematical Society Lecture Series Vol. 16 (Cambridge University Press, Cambridge, England, 2002).

[16] H. P. Bunge, M. A. Richards, and J. R. Baumgardner, Nature (London) 436, 436 (1996).
[17] B. Blankenbach et al., Geophys. J. Int. 98, 23 (1989).

[18] J. Revenaugh and B. Parsons, Geophys. J. R. Astron. Soc. 90, 349 (1987).

[19] G. F. Davies, Dynamic Earth. Plates, Plumes and Mantle Convection (Cambridge University Press, Cambridge, England, 2001).

[20] K. E. Torrance and D. L. Turcotte, J. Fluid Mech. 47, 113 (1971).

[21] F. Dubuffet, D. A. Yuen, and M. Rabinowicz, Earth Planet. Sci. Lett. 171, 401 (1999).

[22] F. Dubuffet, D. A. Yuen, and T. K. B. Yanagawa, Geophys. Res. Lett. 27, 2981 (2000).

[23] T. K. B. Yanagawa, M. Nakada, and D. A. Yuen, Phys. Earth Planet. Inter. 146, 163 (2004).

[24] G. Brandeis and C. Jaupert, Earth Planet. Sci. Lett. 77, 345 (1986).

[25] G. Brandeis and B. D. Marsh, Nature (London) 339, 613 (1989).

[26] J. D. Dufek and G. W. Bergantz, J. Volcanol. Geotherm. Res 143, 113 (2005).

[27] L. G. Mastin, Geochem. Geophys. Geosyst. 3, 1037 (2002).

[28] S. M. Copley, A. F. Giamel, S. M. Johnson, and M. F. Hornbecker, Metall. Trans. 1, 2193 (1970).

[29] J. R. Sarazin and A. Hellawell, Metall. Trans. A 19, 1861 (1988).

[30] M. Ulvrová, S. Labrosse, N. Coltice, P. Raback, and P. J. Tackley, Phys. Earth Planet. Inter. 206-207, 51 (2012).

[31] J. D. Crawford and E. Knobloch, Annu. Rev. Fluid Mech. 23, 341 (1991).

[32] M. Golubitsky and D. G. Schaeffer, Singularities and Groups in Bifurcation Theory, Vol. 1 (Springer, New York, 1985). 
[33] M. Golubitsky, I. Stewart, and D. G. Schaeffer, Singularities and Groups in Bifurcation Theory, Vol. 2, 2nd ed. (Springer, New York, 2000).

[34] M. Field, Trans. Am. Math. Soc. 259, 185 (1980).

[35] D. Ruelle, Arch. Ration. Mech. Anal. 51, 136 (1973).

[36] D. Rand, Arch. Ration. Mech. Anal. 79, 1 (1982).

[37] D. Armbruster, J. Guckenheimer, and P. Holmes, Physica D 29, 257 (1988).

[38] M. Krupa, SIAM J. Math. Anal. 21, 1453 (1990).

[39] J. Guckenheimer and P. Holmes, Math. Proc. Cambridge Philos. Soc. 103, 189 (1988).

[40] S. P. Dawson and A. M. Mancho, Physica D 100, 231 (1997).

[41] M. Golubitsky and I. Stewart, SIAM J. Math. Anal. 17, 249 (1986).

[42] I. Stewart and A. S. Hill, Dyn. Stab. Sys. 6, 267 (1991).

[43] D. Armbruster, J. Guckenheimer, and P. Holmes, SIAM J Appl. Math. 49, 676 (1989).

[44] P. Kolodner, D. Bensimon, and C. M. Surko, Phys. Rev. Lett. 60, 723 (1988).

[45] M. Golubitsky, J. W. Swift, and E. Knobloch, Physica D 10, 249 (1984).

[46] T. Ondarcuhu, G. B. Mindlin, H. L. Mancini, and C. PerezGarcia, Phys. Rev. Lett. 70, 3892 (1993).

[47] G. B. Mindlin, T. Ondarcuhu, H. L. Mancini, C. Perez-Garcia, and A. Garcimartin, Int. J. Bifurcation Chaos 4, 1121 (1994).

[48] D. Krmpotic, G. B. Mindlin, and C. Perez-Garcia, Phys. Rev. E 54, 3609 (1996).

[49] D. Krmpotic, B. Echebarria, and C. Perez-Garcia, Physica D 99, 487 (1997).

[50] P. C. Dauby, P. Colinet, and D. Johnson, Phys. Rev. E 61, 2663 (2000).
[51] P. Assemat, A. Bergeon, and E. Knobloch, Phys. Fluids 19, 104101 (2007).

[52] F. H. Busse, J. Fluid Mech. 72, 65 (1975).

[53] F. H. Busse and N. Riahi, J. Fluid Mech. 123, 283 (1982).

[54] P. Chossat, SIAM J Appl. Math. 37, 624 (1975).

[55] M. Golubitsky and D. G. Schaeffer, Commun. Pure Appl. Math. 35, 81 (1982).

[56] E. Ihrig and M. Golubitsky, Physica D 12, 1 (1984).

[57] J. Schmalzl, M. Breuer, and U. Hansen, Europhys. Lett. 67, 390 (2004).

[58] S. A. van Gilsa and J. Mallet-Paret, Proc. R. Soc. Edinburgh: Sec. A 104, 279 (1986).

[59] L. H. Kellogg and S. D. King, Earth Planet. Sci. Lett. 148, 13 (1997).

[60] V. S. Solomatov and L. N. Moresi, J. Geophys. Res 101, 4737 (1996).

[61] V. S. Solomatov and L. N. Moresi, Geophys. Res. Lett. 24, 1907 (1997).

[62] J. Curbelo and A. M. Mancho, arXiv:1306.2921.

[63] J. Curbelo and A. M. Mancho, Commun. Nonlinear Sci. Numer. Simul. 19, 538 (2014).

[64] F. Pla, A. M. Mancho, and H. Herrero, Physica D 238, 572 (2009).

[65] E. Hairer, S. P. Norsett, and G. Wanner, Solving Ordinary Differential Equations I. Nonstiff Problems (Springer, New York, 2009).

[66] See Supplemental Material at http://link.aps.org/supplemental/ 10.1103/PhysRevE.88.043005 for movies that help to understand the dynamics described in the text.

[67] J. M. Hyman, B. Nicoalaenko, and S. Zaleski, Physica D 23, 265 (1986).

[68] G. I. Sivashinky, SIAM J Appl. Math. 39, 67 (1980). 